F. Ne uner und E. S (iasn ( $^{9}$ ) tatsächlich ein gewisses Diffusionsvermoggen besitzen und da $B$ die Gerbstofflösungen aller Art die verschiedensten Dispersitattsgrade umfassen und die in ihnen enthaltenen Gerbstoffteilchen daher ein entsprechend abgestuftes Diffusionsvermögen zeigen werden. Man kann sich vorstellen, daB die niedrig molekularen bezw. kristalloiden Gerbstoffteilchen leicht in das Innere der Haut und die feinen Hautfibrillen diffundieren, dort unter dem katalytischen Einfluß der Hautfasern durch Kondensationsvorgänge in den hochmolekularen bzw. kolloiden Zustand übergeführt und dann von der Hautfaser adsorbiert werden, während die von vornherein hochmolekularen bezw. kolloiden Gerbstoffteilchen unmittelbar oberflachlich und in grober Verteilung in der Haut zur Adsorption gelangen. Niedrig molekulare Stoffe bezw. deren Lösungen zeigen demnach a is sol c h e keine gerbenden Eigenschaften, sondern nur die an sich hochmolekularen bezw. kolloiden, sowie die nach dem Eindringen in die Haut unter dem EinfluB der Hautfasern entstehenden hochmolekularen bezw. kolloiden Umwandlungsprodukte kristalloider bezw. niedrig molekularer Stoffe. Die Haut wirkt bei der Gerbung in der Hauptsache wohl hauptsächlich in der Weise, dab sie infolge ihres fasrigen Gefüges, ihres gequollenen Zustandes und der

12) Collegium Nr. 404, S. 192 (1910): Der Gerber Nis 850, S. 3 l (1910). dadurch bedingten gröBeren Oberflächenent* wicklung, sowie vielleicht auch katalytisch die zur MolekularvergröBerung und zum Uebergang in den Kolloidzustand führenden chemischen Vorgänge einleitet und begünstigt und in ihrer Eigenschaft als ,organisiertes Gel * die hochmolekularen bezw. kollotden Stoffe durch Adsorption aufnimmt. Schlieblich mub noch angenommen werden, da $B$ die von der Hautfaser adsorbierten Gerbstoffteilchen noch weitere in der Hauptsache irreversible Zustandsänderungen in Sinne der Theorie Sti as ny's durchmachen, wodurch sie zum größten Teil unlöslich werden. Nach dieser Anschauung müBten gerberisch verwertbare Stoffe entweder an sich hochmole. kulare bezw. kolloide Beschaffenheit zeigen oder die Fähigkeit besitzen, beim Eindringen in die Haut unter dem Einflub der Hautfasern in diesen Zustand, soweit er noch nicht vorhanden ist, überzugehen. Chemische Vorgange spielen bei der Gerbung eine Rolle, soweit sie etwa zur Ueberführung der Gerbstoffe in jenen Zustand nötig sind und soweit sie die sekundären Zustandsänderungen der von der Hautfaser adsorbierten Gerbstoffe bedingen. In übrigen kommen nur physikalische Wirkungen und Zustandsänderungen in Betracht. Insbesondere ist die Vereinigung des Gerbstoffes mit der Haut als Adsorptionsvorgang, die entstehende Verbindung als "Adsorptionsverbin* dung aufzufassen.

\title{
Die Kolloidtone, Kaoline und Talke in der Dermatologie.
}

Von Payl Rohland (Stuttgart).

(Eingegaugen am 4. August 1045 )

In der Dermatologie werden jetzt vielfach Präparate mit Bolus, Kaolin, Tierkohle, ferner Kieselsa ure-und Tonerdepräparate in der Kriegsseuchentherapie zur Behandlung von Choleral) und Ruhr, Diphtherie, Fisch- und Fleischvergiftung, ferner von Colitis ulcerosa ${ }^{2}$ ), auch in der $A u$ genheilkund ${ }^{3}$ ) angewand.

2) R. Marcus, Kolloide Kieselsäure und Kieselsturepräparate, derer Anwendung in der inneren Medizin, Chirurgie, Gynakologie und Dermatologie iVerh. d. Ges. d. Naturf. u. Aerzte 1912).

3) R. Albu, Zur Kenntnis det Colitis ulcerosa (Mitt. a. d. Grenzgebieten d. Med. u. Chir. 1914).

7) A. Rosen, Therapeutische Erfahrungen mit Kolloidprảparaten, Salusil, in der Augenheilkunde (Milnch. med. Wochenschrift 6, 1915).
Ihr Heilwert beruht auf ihrer Wasser. imbibitionskraft und ihrem starken Adsorptionsvermögen.

Was die Bolusarten, Talke und Kao. line anbetrifft, so haben meine Untersuch. ungen ) ergeben, dab diese Silikate kolloid veranlagt sind, sie besitzen in lufttrockenem Zustande gewissermaben im latenten Stadium kollo ide Stoffe, die Hydroxyde des Siliziums, Aluminiums und Eisens und organische Stoffe, und bilden sie in Berührung mit Wasser oder Feuchtigkeit aus.

Nicht je de Bolusart ist zu dermatologischen Zwecken verwendbar; diejenigen, die Ánwen1909). 
dung finden können, haben ganz bestimmte Eigenschaften; erstens sie zerfallen im Wasser von selbst zu einem feinen Pulver, zweitens in Berührung mit Hydroxylionen erfolgt eine Ausflockung der Tonteilchen, die mit einer vorübergehenden VolumenvergröBerung verbunden ist, drittens sie adsorbieren kompliziert zusammengesetzte $\mathrm{F}$ a $\mathrm{r}$ st offe, künstliche, wie die Anilinfarbstoffe, ferner pflanzliche und lierische, kolloidgeloste Stoffe und Kolloide, auch starke Gerüche.

Ebenso verhält es sich mit den Kaolinen, von denen sich die gering plastischen nicht eignen.

Ein sehr adsorptionsfuhiger Kolloidton hat der Analyse nach folgende Zusammensetzung:

\begin{tabular}{|c|c|}
\hline $\begin{array}{c}\text { Glühverlust } \\
\mathrm{SiO}_{2} \\
\mathrm{TiO}_{2} \\
\mathrm{SO}_{3} \\
\mathrm{CO}_{2} \\
\mathrm{P}_{2} \mathrm{O}_{\mathrm{g}} \\
\mathrm{Al}_{2} \mathrm{O}_{4} \\
\mathrm{Mn}_{2} \mathrm{O}_{3} \\
\mathrm{CaO} \\
\mathrm{K}_{2} \mathrm{O} \\
\mathrm{Na}_{2} \mathrm{O}\end{array}$ & $\begin{array}{r}8,26 \\
61,30 \\
1,01 \\
1,79 \\
0,74 \\
0,06 \\
17,03 \\
0,14 \\
1,53 \\
1,15 \\
0,71\end{array}$ \\
\hline
\end{tabular}

Aber die kolloidchemische Konstitution der Tone spielt dabei die Hauptrolle.

Beim Kaolin hängt diese auch von seiner Entstehungsweise ab; der von mir benutzte Kaolin, der sich aus Quarzporphyr unter Mitwirkung von Humusstoffen gebildet hatte, besitzt Kolloidstoffe in großer Menge und große Adsorptionsfähigkeit; der Analyse nach hat er folgende Zusammensetzung:

$\begin{array}{ccc}\text { Glühverlust } & 12,67 & \text { Proz. } \\ \mathrm{Si}_{2} & 49,96 " \\ \mathrm{Al}_{2} \mathrm{O}_{3} & 36,33 & . \\ \mathrm{Fe}_{2} \mathrm{O}_{3} & 0,72 & * \\ \mathrm{CaO} & \text { Spuren } \\ \mathrm{Na}_{2} \mathrm{O} & 0,34 . \\ & 00,02 & \text { Proz. }\end{array}$

Kolloidtone und Kaoline sind durch hohen Glahverlust ausgezeichnet, sie enthaiten auch organische, sehr wahrscheinlich kolloide Stoffe, vielleicht Ueberreste einst orga nisierter Materie.

In der Tiefe eines böhmischen Kaolinwerks ist ein Kaolin gefunden worden, der ganz unplastisch was und keine kolloiden Eigen: schaiten hatte.
Bei solchen Adsorptionsversuchen mit Kolloidton und Kaolin muB also darauf geachtet werden, das das Versuchsmaterial die erwähnten Eigenschaften hat.

G. Unna ${ }^{5}$ ) vertritt die Ansicht, daB diese Adsorptionen nicht auf die Kolloidstoffe zurückzuführen sind, er meint. daß g eglühter Kaolin keine organischen Kolloide enthalten könne und anorganische in sehr geringer Menge und doch adsorbiert.

Das erstere ist richtig, das zweite nicht. Auch der geglühte Kaolin bildet in Berührung mit Feuchtigkeit oder Wasser genügend anorganische Kolloide, um diese Adsorptionen ausüben zu können.

Dann hat Unna auch kristalloide Stoffe, wie Magnesiumkarbonat, angewandt; hier handelt es sich aber garnicht um eigentliche Adsorptionen; ein bedeutsames Kennzeichen dieser ist, dab ein kompliziert zusammengesetzter Farbstoff, wie ein Aniliniarbstoff, der von Kolloidton oder Kaolin adsorbiert worden ist, von Wasser, auch von heibem, nicht wieder herausgewaschen werden kann.

Besonders interessant ist die Adsorption dieser kolloid veranlagten Sillkate gegenüber starken Gerüchen; schüttelt man Kolloidton mit einer Lösung von $\mathrm{E}$ is ens accharat, so geht der den Tonen eigentümliche Geruch und Geschmack auf das Eisensaccharat über, während der Ton dann sü̈ schmeckt.

Schüttelt man Kaolin mit Ammoniaklösung, so hat diese den Geruch ron Kuolin angenommen, während der Kaolin nach Ammoniak. riecht. Es findet also ein Austausch statt.

Talk, in Berührung mit $\mathrm{Ch}$ lorkalk, hatte in stärkstem Maße den Chlorgeruch angenommen. Auch Wohlgerüche werden übertragen $\left.^{6}\right)$.

Um ja u chige Gerüche zu entfernen, empfiehlt $K . O$ pit $z^{7}$ ) zehnprozentiges $\mid \mathbf{c h t}$ h y ol. kieselsà repulver.

Da der Kaolin im Gegensatz zu den Kolloidtonen am wenigsten Verunreinigungen enthält, die Störungen verursachen könnten, so ist am besten ein sehr plastischer, kolloidreicher Kaolin, z. B. vom Kaolinwerk Spergau in Magdeburg in der Dermatologie zu verwenden.

5).G. Unna, Dic Kaolinglyzerinpaste in der Dermatologie (Med. Klinik 41 und 42, 1913).

6) Vgl. P. Rohland, Kolloidchemic und Parfümeriefabrikation (Deutsche Parfümeriezeitung Nr. 6, $1915)$.

5) R. Marcus, Verh. d. Ges. d. Naturf. u. Aerzte 19!2, loc, cit. 
Die Prüfung, ob sich ein Kaolin, Talk oder Kolloidton hierzu eignet, kann bequem durch eine quantitative Bestimmung der Kolloide mit Hilfe der Adsorption durch einen kompliziert zusammengesetzten Farbstoff, z. B. Methylenblau, ermittelt werden.

$10 \mathrm{~g}$ Bolus oder Kaolin werden mit $100 \mathrm{ccm}$ der Farbstofflösung bekannten Gehalts geschüttelt, mehrere Stunden absitzen gelassen, filtriert und durch eine kolorimetrische Bestimmung der Farbstoffgehalt ermittelt.

Die Differenz des ursprünglichen Gehalts der Farbstofflösung und des Gehalts des Filtrats gibt die vom Kaolin adsorbierte Farbstoffmenge an, und nach dem Grad seiner Adsorptionsfähigkeit läBt sich auch der Grad seiner Verwendbarkeit in der Dermatologie beurteilen.

Die zu den Versuchen benutzten T.alke ${ }^{8}$ ) hatten nach der Analyse folgende Zusammensetzung :



Die Talke, saure Salze der Metakieselsäure, $\mathrm{Mg}_{3} \mathrm{H}_{2}\left(\mathrm{SiO}_{5}\right)_{4}$ werden schon bei gewöhnlicher Temperatur hydrolytisch gespalten, noch stärker bei $50^{\circ}$, sie bilden in Berührung mit Wasser kolloide Stoffe, die Hydroxyde des Siliziums, Magnesiums, Aluminiums und Eisens und haben daher eine sehr starke Adsorptionsfähigkeit.

Werden auBer dem Wasser ein paar Tropfen verdünnte $S$ alzsäure zu dem Talk gesetzt, so tritt ein eigentümliches $S c h a ̈ u m e n$ auf, in ähnlicher Weise wie beim EiweiB.

Bezüglich der Kieselsäure sind noch einige Unstimmigkeiten vorhanden in Hinsicht

8) Bezogen von der Firma Bernfeld \& Rosenberg in Wien. auf ihre Adsorptionsfăhigkeit gegenüber Toxinen. So ist gefunden worden, dab es eine chemisch reine Kieselsäure gibt, die Diphtherietoxin adsorbiert, aber. nicht Diphtherieantitoxin, und eine andere, ebenfalls chemisch reine, die dieses $n i c h t$ adsorbiert, hingegen Diphtherietoxin. Die zweite Kieselsäure ist auf elektroosmotl. schem Wege gewonnen worden, die erste auf rein chemischem. Nach Versuchen von $E$. $\mathrm{Z} u n z^{9}$ ) adsorbiert die chemisch reine Kieselsäure Antitoxin von Tetanus und Antilysin von Cobra. die osmotische aber nicht.

Die beiden chemisch reinen Kieselsäurepräparate $\mathrm{s} c \mathrm{~h}$ e in e $\mathrm{n}$ ganz verschieden in thren Adsorptionseigenschaften zu sein.

Auch $\mathrm{Ki}$ eselgur hat nicht die geringste Fähigkeit, Diphtherietoxin zu adsorbieren.

Die Erklärung hierfür dürite darin zu suchen sein, daß in den Adsorptionsvermogen der einzelnen Kieselsäuren gegenüber den Toxinen je nach ihrer Struktur eine Abstufung stattfindet; bei keiner aber ist die Adsorptionsfähigkeit gleich Null, sondern nur sehr klein.

Etwas Aehnliches ist auch bei den Kolloidtonen $z u$ beobachten; sie adsorbieren zwar alle Anilinfarbstoffe, aber je nach ihrer Konstitution in verschiedenem MaBe; und dann wieder die blauen viel besser als die gelben ${ }^{10}$ ).

Nach einem neuen Verfahren ist es jetzt gelungen, auch $\mathrm{Bals}$ a me, z. B. Perubalsam, von Kieselsäure usw. adsorbieren zu lassen; trotadem adsorbieren diese Präparate noch Toxine.

Nach meinen Versuchen hatten von den Präparaten der Salusitwerke in Mainz-Kastel bzw. Frankfurt a. M. die größte Adsorptionsfähigkeit gegenüber kompliziert zusammengesetzten Farbstoffen diejenigen, die aus Kieselsäuregallerte, elektroosmotischer Kieselsåure, chemisch gefällter reiner Kieselsäure und Tierk ohle zusammengesetzt waren.

9) E. Zun z, Zeitschr. f. Immun.-Forsch. 19, Heft 3

10) Vgl. P. Rohland, Die Adsorptionsfahigkeit des Kolloidtans (Koll.-Zeitschr. 16, 16, 1915). 